\title{
Analyst
}

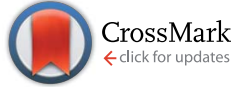

Cite this: Analyst, 2015, 140, 107

Received 6th October 2014

Accepted 24th October 2014

DOI: $10.1039 / c 4 a n 01798 f$

www.rsc.org/analyst

\section{Development of Transmission Raman Spectroscopy towards the in line, high throughput and non- destructive quantitative analysis of pharmaceutical solid oral dose $\uparrow$}

\begin{abstract}
Julia A. Griffen, ${ }^{a}$ Andrew W. Owen ${ }^{a}$ and Pavel Matousek ${ }^{\star a b}$
Transmission Raman spectroscopy (TRS) is a recently introduced analytical technique to pharmaceutical analysis permitting volumetric sampling by non-destructive means. Here we demonstrate experimentally, for the first time, the enhanced speed of quantification of pharmaceutical tablets by an order of magnitude compared with conventional TRS. This is achieved using an enhancing element, "photon diode", avoiding the loss of laser photons at laser coupling interface. The proof-of-concept experiments were performed on a complex mixture consisting of 5 components ( 3 APIs and 2 excipients) with nominal concentrations ranging between 0.4 and $89 \%$. Acquisition times as short as $0.01 \mathrm{~s}$ were reached with satisfactory quantification accuracy for all the sample components. Results suggest that even faster sampling speeds would be achievable for components with stronger Raman scattering cross sections or with higher laser powers. This major improvement in speed of volumetric analysis enables high throughput deployment of TRS for in line quality control applications within the batch or continuous manufacturing process and facilitating non-destructive analysis of large fractions.
\end{abstract}

\section{Introduction}

The most widely used analytical method for the determination of assay, potency or content uniformity of pharmaceutical solid oral dose forms is High Performance Liquid Chromatography (HPLC). Although accurate and highly sensitive, this technique has several disadvantages: it is destructive, requires sample preparation and consumables, analysis times are typically longer than the batch manufacturing time of the product and consequently has limited throughput. HPLC is therefore unsuitable for routine analysis of large proportions of manufactured batches.

The advantages of sampling larger proportions of the total batch size include: meeting regulatory demands, reduction in batch rejects and higher confidence that the release product is of correct parameters. Analysis has to be with a non-destructive technique otherwise the cost of analysis would out way these benefits.

Transmission Near Infrared (NIR) spectroscopy is a nondestructive technique that can, in some cases, quantify active ingredients in tablets and capsules with adequate accuracy. Analysis times are, typically, in the orders of tens of seconds.

${ }^{a}$ Cobalt Light Systems Ltd, 174 Brook Drive, Milton Park, Abingdon, Oxfordshire OX14 $4 S D, U K$

${ }^{b}$ Central Laser Facility, Research Complex at Harwell, STFC Rutherford Appleton Laboratory, Harwell, Oxford OX11 0QX, UK. E-mail: Pavel.Matousek@stfc.ac.uk

$\dagger$ Electronic supplementary information (ESI) available. See DOI: 10.1039/c4an01798f
Recently a new potent volumetric analytical tool has emerged in this area - Transmission Raman Spectroscopy (TRS). ${ }^{\mathbf{1 - 3}}$ To date it has been used in several studies performed at line (i.e. off line, next to the production line and separately from it) demonstrating capability to predict tablets and capsule components in quantitative manner with required accuracy. ${ }^{4-8}$ The method was shown to provide volumetric sampling capability and inherently considerably higher sampling speeds than NIR. Typical TRS sampling times demonstrated range from seconds to tens of seconds per tablet or capsule when deployed in conventional form (although faster speeds are achievable with strong Raman scatterers or at high concentrations).

In this paper, we demonstrate, for the first time, the capability of TRS to provide an order of magnitude enhanced sampling speeds in quantitative TRS analysis than achievable with conventional TRS technique. This is accomplished using a recently introduced signal enhancing 'photon diode' that is capable of increasing Raman light throughput at around tenfold in a passive manner. ${ }^{9}$ Although the signal enhancement characteristics have been described the technique has not been demonstrated in quantitative analysis. As the signal-to-noise ratio (SNR) presents one of the limiting factors of conventional TRS methods in rapid quantitative analysis, the signal enhancement due to the photon diode, which also leads directly to SNR improvement, has a potential to translate itself directly into the improved precision of quantification. This hypothesis has been experimentally tested in the research presented here. 
In essence, the photon diode is a 'unidirectional' mirror permitting the transfer of photons from one side and acting as a reflector for photons impacting on it from the other side. ${ }^{9}$ It is located in close proximity to sample placed directly over the laser illumination zone to prevent the loss of diffusely scattered photons from sample surface. As this loss can typically be substantial $(>90 \%$ of photons can escape by this mechanism from sample) its prevention leads to much higher coupling efficiency of laser photons into the sample.

The photon diode itself is fabricated as a multilayer dielectric optical bandpass or edge filter and has identical properties. Its unidirectional property stems from the generic angular

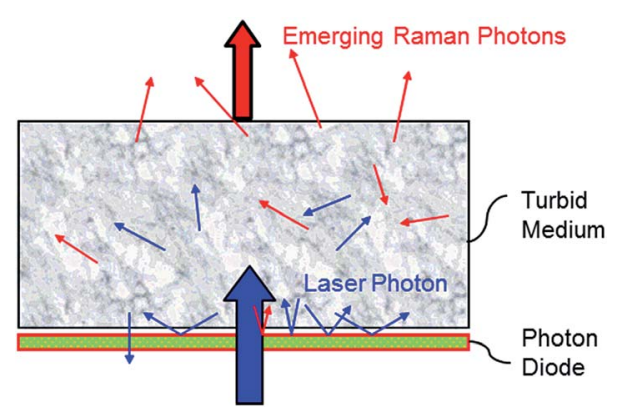

Fig. 1 Schematic of a photon diode or 'unidirectional mirror'. Tablet is placed directly on top of photon diode. Any laser photons which are re-radiated backwards from the tablet surface are reflected back into the sample. The net effect of increased laser photons into the sample results in an increase of emerging Raman photons. properties of dielectric filters for which the spectral profile shifts to shorter wavelengths as photons impact them at greater angles further away from normal incidence. The substantially increased coupling of laser radiation naturally leads to greater Raman signal and improved SNR of the detected Raman spectrum. Fig. 1.

An alternative enhancing concept to photon diode is a hemispherical mirror ${ }^{\mathbf{1 0}}$ which too could be used to recycle diffusely reflected laser photons back into the sample to enhance Raman signal efficiency.

\section{Experimental}

The TRS spectra were collected using a commercial TRS instrument (TRS100, Cobalt Light Systems Ltd, Oxfordshire, UK). The system operates at $830 \mathrm{~nm}$ laser excitation wavelength.

The acquisition parameters employed a $4 \mathrm{~mm}$ laser illumination spot diameter and the power at sample was set to

Table 2 Acquisition parameters for each scan time

\begin{tabular}{lllll}
\hline Method & $\begin{array}{l}\text { Laser } \\
\text { power }(\mathrm{mW})\end{array}$ & $\begin{array}{l}\text { Sub-exposure } \\
\text { time }(\mathrm{s})\end{array}$ & $\begin{array}{l}\text { Number of } \\
\text { accumulations }\end{array}$ & $\begin{array}{l}\text { Total exposure } \\
\text { time }(\mathrm{s})\end{array}$ \\
\hline 1 & 200 & 0.01 & 1000 & 10.00 \\
2 & 200 & 0.01 & 100 & 1.00 \\
3 & 200 & 0.01 & 10 & 0.10 \\
4 & 200 & 0.01 & 1 & 0.01
\end{tabular}

Table 1 Calibration DoE concentration ranges of components

\begin{tabular}{|c|c|c|c|c|c|}
\hline Sample & Phenylephrine \% w/w & Caffeine $\% \mathrm{w} / \mathrm{w}$ & Mag stearate \% w/w & Tablettose \% w/w & Paracetamol \% w/w \\
\hline 1 & 1.22 & 5.19 & 1.29 & 10.03 & 82.27 \\
\hline 2 & 1.18 & 1.36 & 0.46 & 8.19 & 88.81 \\
\hline 3 & 0.51 & 1.50 & 2.28 & 9.55 & 86.15 \\
\hline 4 & 0.39 & 9.07 & 0.95 & 7.49 & 82.10 \\
\hline 5 & 1.94 & 3.26 & 1.88 & 13.43 & 79.48 \\
\hline 6 & 0.96 & 8.42 & 1.28 & 7.98 & 81.36 \\
\hline 7 & 2.27 & 5.01 & 0.93 & 10.99 & 80.80 \\
\hline 8 & 1.31 & 3.33 & 0.84 & 9.29 & 85.23 \\
\hline 9 & 0.78 & 3.55 & 1.63 & 10.46 & 83.57 \\
\hline 10 & 0.86 & 7.09 & 2.20 & 11.97 & 77.88 \\
\hline 11 & 1.67 & 8.57 & 1.71 & 12.24 & 75.80 \\
\hline 12 & 2.10 & 6.91 & 1.37 & 13.52 & 76.10 \\
\hline 13 & 1.73 & 5.26 & 2.21 & 15.48 & 75.32 \\
\hline 14 & 1.37 & 8.49 & 2.20 & 11.46 & 76.49 \\
\hline 15 & 2.03 & 8.86 & 0.50 & 10.26 & 78.35 \\
\hline 16 & 2.02 & 1.73 & 1.67 & 11.68 & 82.90 \\
\hline 17 & 0.56 & 6.71 & 0.49 & 5.36 & 86.88 \\
\hline 18 & 1.70 & 2.33 & 1.33 & 11.59 & 83.06 \\
\hline 19 & 0.50 & 5.43 & 1.57 & 8.98 & 83.52 \\
\hline 20 & 1.32 & 6.71 & 1.77 & 10.94 & 79.27 \\
\hline 21 & 1.73 & 6.82 & 0.84 & 8.54 & 82.08 \\
\hline 22 & 1.74 & 3.65 & 0.55 & 8.53 & 85.53 \\
\hline 23 & 0.87 & 1.78 & 0.80 & 7.24 & 89.31 \\
\hline 24 & 0.42 & 3.39 & 1.19 & 5.55 & 89.44 \\
\hline Average & 1.3 & 5.2 & 1.3 & 9.8 & 82.4 \\
\hline Min & 0.4 & 1.4 & 0.5 & 5.2 & 75.3 \\
\hline Max & 2.3 & 9.1 & 2.3 & 15.5 & 89.4 \\
\hline
\end{tabular}


$200 \mathrm{~mW}$. A Peltier cooled CCD detector was used to detect the Raman spectra (Andor Technology, DU420A-BR-DD, $1024 \times 256$ pixels, $-75{ }^{\circ} \mathrm{C}$ ). The acquisition time was varied systematically from 0.01 to $10 \mathrm{~s}$. Multivariate modelling was performed using Solo (Eigenvector Research Inc., Wenatchee, WA, US).

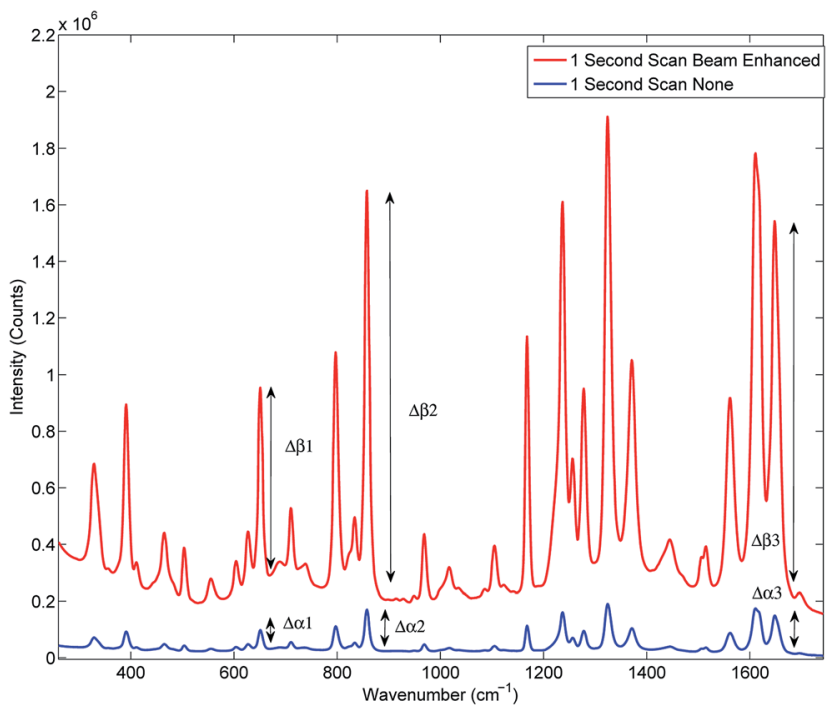

Fig. 2 Signal enhancement of the average spectra of the entire data set scanned for 1 second with (top trace) and without beam enhancer (bottom trace). Signal enhancement of 9.9 has been measured.
The photon diode comprised of a $25 \mathrm{~mm}$ diameter dielectric bandpass filter (Iridian) centered at $830 \mathrm{~nm}$ with a bandwidth of $2.2 \mathrm{~nm}$ (FWHM) and transmission of $>90 \%$ at the central wavelength.

Tablets were prepared from individual constituent powders weighed and mixed by hand, ground with a pestle and mortar and compressed using $8 \mathrm{~mm}$ flat surface tablet die in a bench top vice into two tablets per sample. The average mass of the tablets was $\sim 200 \mathrm{mg}$ with a range between 180-225 $\mathrm{mg}$. The average thickness of the tablets was $3.3 \mathrm{~mm}$ with a range $3.0-3.7 \mathrm{~mm}$.

The basic mixture approximated commonly available overthe-counter cold \& flu remedy tablets. It consisted of three active pharmaceutical ingredients (APIs): paracetamol (Sigma-Aldrich $\sim 85 \% \mathrm{w} / \mathrm{w}$ ), caffeine (Sigma-Aldrich, $\sim 4 \% \mathrm{w} / \mathrm{w}$ ), phenylephrine (Sigma-Aldrich $\sim 1 \% \mathrm{w} / \mathrm{w}$ ); and two excipients: tablettose ${ }^{\circledR}$

Table 3 Beam Enhancement calculation (1 second scan time data)

\begin{tabular}{lllr}
\hline Peak & Beam enhanced $\Delta \beta$ & None $\Delta \alpha$ & $\Delta \beta / \Delta \alpha$ \\
\hline 1 & 650901 & 64695 & 10.1 \\
2 & 1417197 & 143841 & 9.9 \\
3 & 1305761 & 133168 & 9.8 \\
& Average enhancement & & 9.9
\end{tabular}
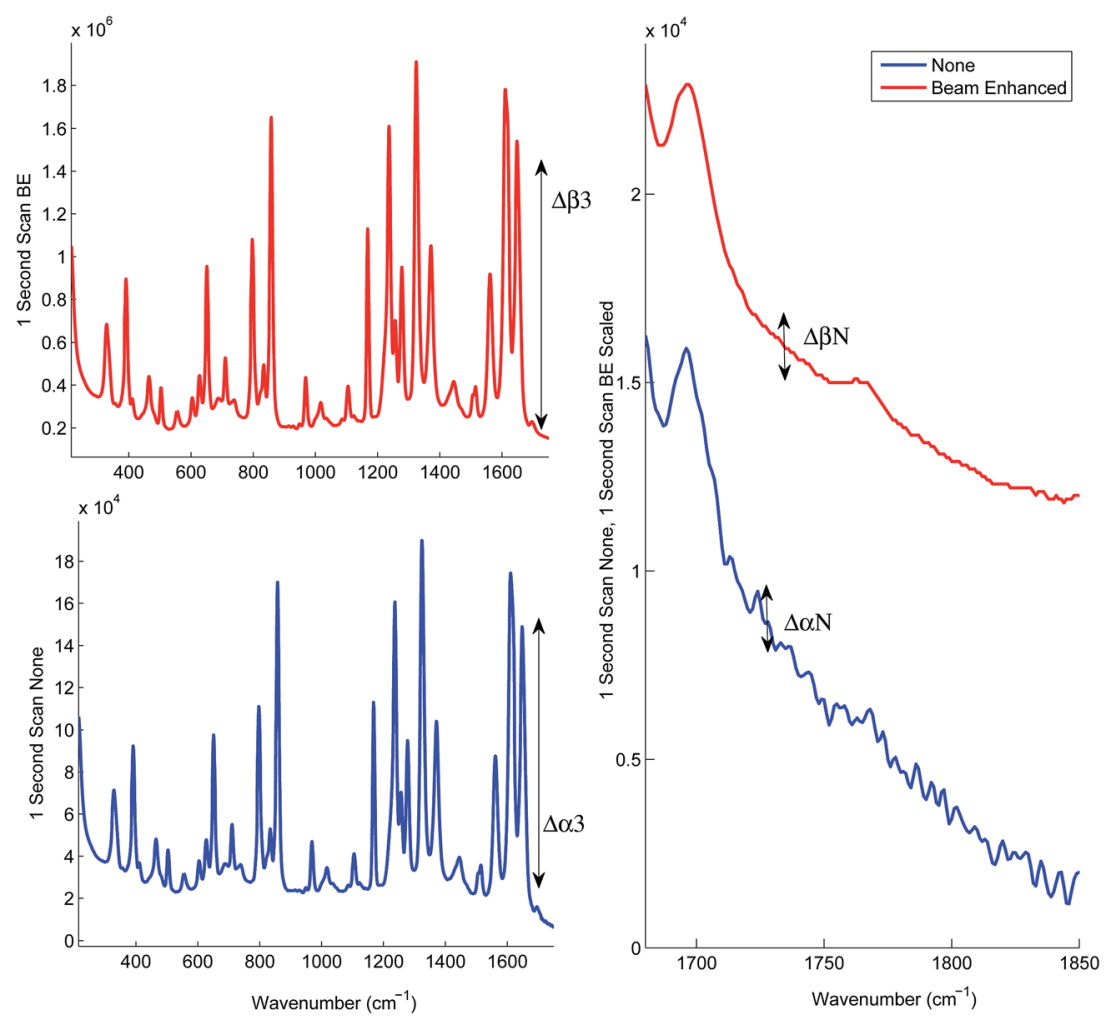

Fig. $31 \mathrm{~s} \mathrm{scan}$ time. The beam enhanced spectra have been reduced and scaled to the same relative intensity as the 'None'. Signal-to-noise analysis of the TRS spectra with and without the photon diode was performed to illustrate the improvement of SNR accompanying the boost of the signal intensity with photon diode. 
[compressible lactose powder] (Meggle $\sim 10 \% \quad \mathrm{w} / \mathrm{w}$ ) and magnesium stearate (Sigma-Aldrich $\sim 1 \% \mathrm{w} / \mathrm{w}$ ).

The design of a calibration set followed a first-order (linear) 5 concentration level orthogonal design ${ }^{\mathbf{1 1}}$ uniformly covering the respective concentration ranges. Paracetamol, the bulk constituent, was used as the filler. Details of the individual 25 samples are shown in Table 1.

A total of 768 spectra were collected for analysis by scanning all 48 tablets from either face, being placed on photon diode band pass filter or on a bare silica plate and scanned at each of the four acquisition parameters detailed in Table 2. (24 samples $\times 2$ tablets $\times 2$ faces $\times 2$ filter options $\times 4$ methods $=768$ spectra). Note that tablets were not moved between changing the acquisition parameters to minimise variation in tablet placement.

\section{Results and discussion}

Prior to the quantification measurements we first characterised the enhancement effect of photon diode across the sample range by comparing the average spectra of the entire data set, and its influence on the signal-to-noise ratio in TRS spectra.

Fig. 2 and Table 3 illustrates the Raman intensity enhancement effect attained using a photon diode compared to a tablet placed on a bare silica plate acting as a reference for the 1 second total scan time data set. An overall Raman signal intensity enhancement of approximately 10 has been achieved.

Fig. 3 illustrates the improvement of SNR in TRS spectra upon the deployment of photon diode. The spectra are acquired under the same acquisition time (1 s). In the absence of any other source of noise the signal-to-noise ratio of TRS spectra is limited by the photon shot noise generated by the signal itself (i.e. Raman and any fluorescence background if present). The SNR is therefore theoretically expected to improve with the square root of enhancement factor, i.e. 3.3 times. ${ }^{12}$ From the statistical analysis of noise within the region $1730-1755 \mathrm{~cm}^{-1}$ we estimate the measured improvement in signal to noise experimentally to be 5.6. A higher than theoretical value indicates an additional noise contribution originating possibly from the detection instrument (e.g. due to CCD detector read out noise). Similarly high SNR improvement factors with photon diode were estimated for the Raman spectra acquired at the other acquisition times.

In the next series of experiments the sample set was subjected to TRS analysis performed with and without the photon diode. The tests were carried out separately at acquisition times of $10.00,1.00,0.10$ and 0.01 s. Fig. 4 shows the resulting TRS spectra of the average spectra for entire data set acquired at the
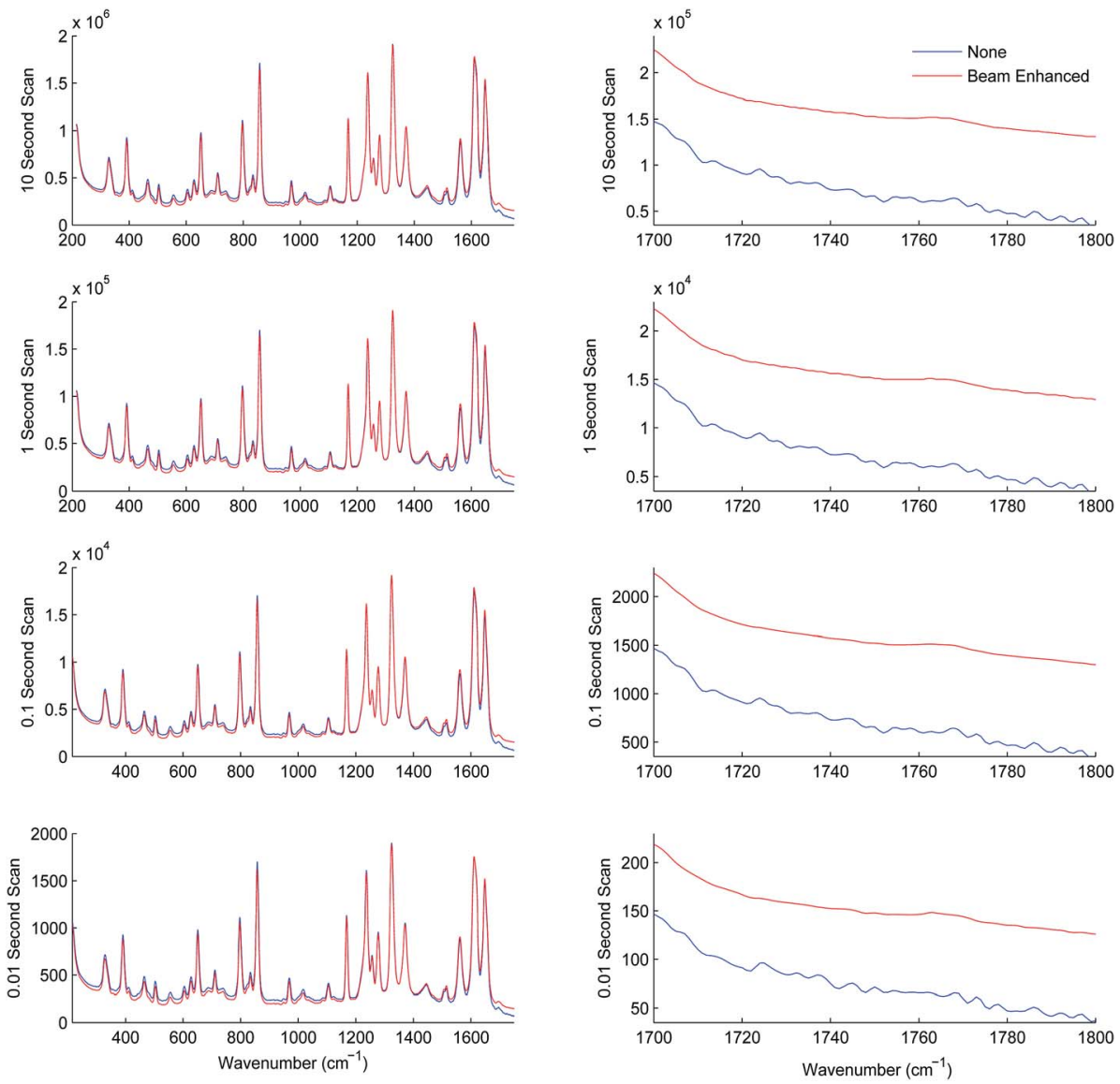

Fig. 4 TRS spectra of the average spectra of the entire data set measured at four different acquisition times with and without photon diode. Beam enhanced spectra have been reduced in scale to the same relative intensity as 'None' to highlight enhancement in signal to noise ratios. Especially observable over the relatively blank regions $1700-1800 \mathrm{~cm}^{-1}$. 
different acquisition times, with and without photon diode. Visually the enhancement in signal to noise is uniform across each of the time points. Table 4 summarises the enhancement factor and enhancement in SNR for each of the acquisition parameters. Statistically there is consistent enhancement in signal across the acquisition time points. The SNR improvement factor varied between 4.8 to 5.9 .

Next we subjected the calibration data sets to PLS analysis and recovered RMSECV for each experimental configuration, acquisition parameters and beam enhancement. The results are depicted in Fig. 5 and 6. Further information can be found in the ESI. $\dagger$

From the analysis and consideration of the statistical outputs of the PLS models, $R^{2}$, RMSECV values and visualised fit and trending of these values (Fig. 5 and 6), we have found consistently improved results were obtained with the photon diode permitting good quantification performance to be attained down to a level of $0.01 \mathrm{~s}$ acquisition times. Overall the use of photon diode alone clearly lowered the required acquisition times down approximately by an order of magnitude for achieving the same level of quantification accuracy as without the photon diode.

Due to the sample set containing different constituents at varying concentrations we were able to observe the improvement of the quantification of individual constituents at different concentration levels as a function of scan time and beam enhancer. It would appear that the improvement of the

Table 4 Signal to noise calculations for each of the acquisition parameters

\begin{tabular}{ll}
$\begin{array}{l}\text { Signal } \\
\text { enhancement }\end{array}$ & $\begin{array}{l}\text { Overall SNR } \\
\text { improvement }\end{array}$ \\
$\Delta \beta 3 \div \Delta \alpha 3$ & SNR $\beta \div$ SNR $\alpha$ \\
\hline 10 & 4.8 \\
10 & 5.6 \\
10 & 5.9 \\
10 & 4.9
\end{tabular}

$\begin{array}{lll}\text { 10 Second scan time } & 10 & 4.8 \\ \text { 1 Second scan time } & 10 & 5.6 \\ 0.1 \text { Second scan time } & 10 & 5.9 \\ 0.01 \text { Second scan time } & 10 & 4.9\end{array}$

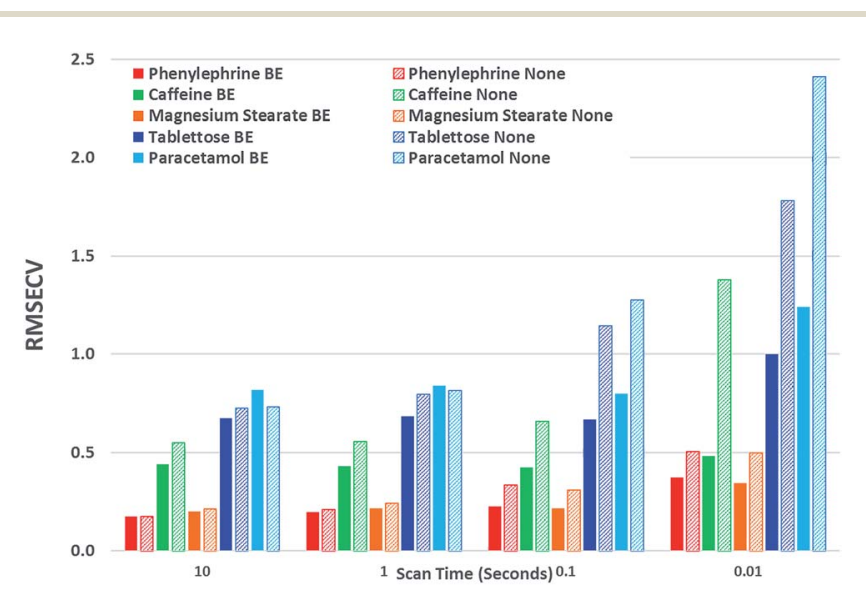

Fig. 5 Graphical summary of RMSECV Values.

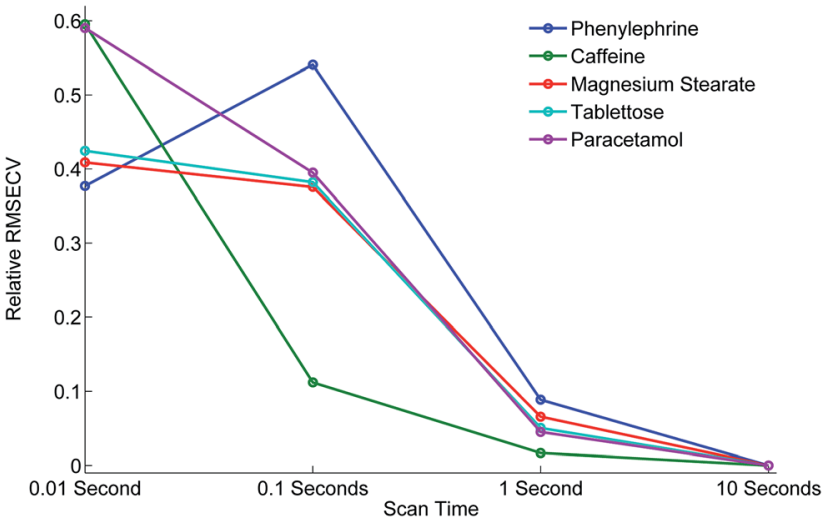

Fig. 6 Relative improvement in RMSECV, scaled to the same $Y$ axis for ease of comparison.

quantification of individual constituents due to the engagement of photon diode vary differently, for instance it depends on the inherent intensity and Raman scattering properties of the constituent and the relative proportion or concentration present in the sample.

For example, caffeine gives an intense Raman signal and at a nominal concentration of $5 \%$, the use of the photon diode gives improved model statistics across all scan times. Conversely, paracetamol, although an excellent Raman scatterer, at 85\% nominal concentration the beam enhancer does not improve model statistics at longer scan times, conversely model statistics slightly worsen. It could be suggested that under the experimental conditions, with these particular samples we have reached the optimal model statistics for paracetamol, and no further improvement can be made by scanning the samples for longer, or using beam enhancers. This is presumably due to some other effect independent of SNR within Raman spectra, which clamps the achievable RMSECV values at such longer acquisition times. We speculate that these clamping effects could for example be due to remaining heterogeneity of tablets not removed fully by the TRS method that becomes a dominant factor below certain level of SNR within spectra or due to residual errors in weighing and blending individual tablet components.

Considering relative improvement in RMSECV values, caffeine relative improvement at $0.01 \mathrm{~s}$ scan times is steep, suggesting that even faster scan times and relative improvements in RMSECV can be achieved using the beam enhancer technology.

Overall it would seem that beam enhancers have the greatest and most noticeable improvement at short acquisition times. This is expected as SNR due to photon shot noise within spectra becomes the limiting factor for achievable quantification in this regime in contrast with longer acquisition times where other effects such as tablet heterogeneity or sample preparation accuracy may be the limiting factors for reaching lower RMSECV values. The relative enhancement is also dependent on the inherent nature of the constituent being analysed. 


\section{Conclusions}

The study demonstrated the feasibility of dramatically shortening the acquisition times (by an order of magnitude) by using a photon diode signal enhancing element. Acquisition times as short as $0.01 \mathrm{~s}$ per tablet were sufficient to achieve a good degree of quantification with API at concentrations ranging from 0.4 to $89 \%$. Still higher sampling speeds would be achievable for higher API concentrations or API with stronger Raman scattering cross sections than those used here. The method paves the way for the next generation of TRS analytical tools permitting the use of this technology in line in manufacturing environment for fast non-destructive analysis of large portions of manufactured products than possible to date.

\section{References}

1 B. Schrader and G. Bergmann, Fresenius' J. Anal. Chem., 1967, 225, 230-247.

2 P. Matousek and A. W. Parker, Appl. Spectrosc., 2006, 60, 1353-1357.
3 P. Matousek and A. W. Parker, J. Raman Spectrosc., 2007, 38, 563-567.

4 J. Johansson, A. Sparen, O. Svensson, S. Folestad and M. Claybourn, Appl. Spectrosc., 2007, 61, 1211-1218.

5 C. Eliasson, N. A. Macleod, L. C. Jayes, F. C. Clarke, S. V. Hammond, M. R. Smith and P. Matousek, J. Pharm. Biomed. Anal., 2008, 47, 221-229.

6 M. D. Hargreaves, N. A. Macleod, M. R. Smith, D. Andrews, S. V. Hammond and P. Matousek, J. Pharm. Biomed. Anal., 2010, 54, 463-468.

7 A. Aina, M. D. Hargreaves, P. Matousek and J. C. Burley, Analyst, 2010, 135, 2328-2333.

8 M. Fransson, J. Johansson, A. Sparen and O. Svensson, J. Chemom., 2010, 24, 674-680.

9 P. Matousek, Appl. Spectrosc., 2007, 61, 845-854.

10 M. J. Pelletier, Appl. Spectrosc., 2013, 67, 829-840.

11 R. Brereton, Analyst, 1997, 122, 1521-1529.

12 P. Matousek, M. Towrie and A. W. Parker, J. Raman Spectrosc., 2002, 33, 238-242. 